\title{
Metastasia Lesion of Gestation Trophoblastic Tumor in Abdominopelvic Cavity Diagnosed by Sonographic and Doppler Imaging
}

\author{
Guozhen Yan*, Yanfen Yuan, Yang Liu, Yunmei Lv, Aihua Li \\ Department of Ultrasound Diagnostics, The First Affiliated Hospital of the Baotou Medical College, \\ Baotou, China \\ Email: ${ }^{*}$ YGZ670708@163.com
}

Received 4 February 2015; accepted 24 March 2015, published 27 March 2015

Copyright ( 2015 by authors and Scientific Research Publishing Inc.

This work is licensed under the Creative Commons Attribution International License (CC BY). http://creativecommons.org/licenses/by/4.0/

(c) $\underset{\mathrm{EY}}{\mathrm{C}}$ Open Access

\section{Abstract}

Objective: The purpose of this study was to review clinical data of metastasia lesion of gestation trophoblastic tumor (GTT) in abdominopelvic cavity by color Doppler ultrasound. Subjects and Methods: A retrospective analysis of 13 cases of GTT in China was performed. Clinical appearances, serum human chorionic gonadotropin (hCG) levels, sonographic findings, Doppler waveforms, and patient outcomes were followed up. Color Doppler ultrasound was performed to diagnose the presence of GTT, detect metastasia lesion in abdominopelvic cavity, assess disease recurrence, and monitor the efficacy of chemotherapy. Results: Of the 13 patients with GTT, 4 had choriocarcinoma which 3 cases occurred after an abortion, 1 after a year of dilation and suction evacuation for the hydatidform mole. Metastasia lesion in abdominopelvic cavity was detected by color Doppler ultrasound in all cases of choriocarcinoma, among which the metastasia lesion were found at uterine fundus, near uterus, colon and cystic vascular space near the uterus, repectively. 9 cases with an invasive hydatidiforn mole (IHM) occurred after one to three months of dilation and suction evacuation for the hydatidform mole, consistant with the hCG levels markedly elevating. Metastasia lesion in abdominopelvic cavity was also detected by color Doppler ultrasound besides invasive lesion in uterine muscular layer in all cases of IHM, among which 3 cases showed metastasia lesion in cystic vascular space near the uterus, 2 cases were detected in side wall of pelvic cavity, while the rest were found in abdominal cavity, right kidney, colon and near uterus, respectively. Meanwhile, the image of metastasia lesion with IHM was similar to choriocarcinoma's. And the reticular structure, the cystic vascular space and solid lesion may be showed by ultrasound. The metastasia lesion had abundant flow. Doppler waveforms showed resistive indices of 0.34 (SD 0.07) for metastasia lesion. There was the same lower- impedance as invasive lesion in

\footnotetext{
*Corresponding author.
}

How to cite this paper: Yan, G.Z., Yuan, Y.F., Liu, Y., Lv, Y.M. and Li, A.H. (2015) Metastasia Lesion of Gestation Trophoblastic Tumor in Abdominopelvic Cavity Diagnosed by Sonographic and Doppler Imaging. Open Journal of Clinical Diagnostics, 5, 24-31. http://dx.doi.org/10.4236/ojcd.2015.51005 
uterine muscular layer. Except an IHM with the renal metastasia lesion having been followed, abnormal sonographic and Doppler findings in other metastasia disease all disappeared after surgical operation or chemotherapy were successful. Conclusions: Sonography and Doppler imaging were helpful in detecting metastasia lesion in abdominopelvic cavity, and in following the effectiveness of chemotherapy. And it should be taken full examination for GTT by Color Doppler ultrasound in order to avoid mistake.

\section{Keywords}

Gestation Trophoblastic Tumor, Color Doppler Ultrasound, Metastasia Lesion

\section{Introduction}

Gestation trophoblastic tumor (GTT) is a group of trophoblastic diseases rooting in embryo, which often subdivided into IHM, choriocarcinoma and placental site trophoblastic tumor. All these show abnormal trophoblastic proliferation histologically [1] [2]. Because of invasive behavior of GTT, metastases always occur. The site of GTT with metastasia lesion may be lung, brain, bladder, right ventricle, testis and so on [3]-[8]. Rare sites of metastases to the spinal cord and paraspinous tissues have also been reported [9]. A review of the literature has shown that most metastasia lesions were diagnosed with the aid of computed tomography and X-Ray. The metastasia lesion of GTT in abdominopelvic cavity is rare reported only by ultrasound.

We have been studying gestation trophoblastic disease for a long time, and articles about 12-year experience in China have been published [10] [11]. This study emphasizes to detect metastasia lesion of GTT in abdominopelvic cavity by abdominopelvic sonography in order to avoid missing and making mistakes. The study is done after agreement from the local ethics committee and with the patients' informed consent. The present study is performed in accordance with the ethical standards formulated in the 1975 version of the Declaration of Helsinki.

\section{Materials and Methods}

Clinical histories, sonographic images, and Doppler waveform of 13 cases of GTT diagnosed and treated at the first affiliated hospital of Baotou medical college in China between 2000 and 2006 were evaluated retrospectively. In the meantime, the comprehensive modern hospital has received a total of 93 cases of gestational trophoblastic tumor patients. Choriocarcinoma was diagnosed in 4 women and IHM was diagnosed in 9.

The age of patients with GTT ranged from 21 to 46 years. The mean age was 34 years (SD 7.3 years). The parity history and evident symptom were recorded. Serum hCG level was evaluated in all patients, too.

In addition, all patients were studied by abdominopelvic sonography, which include color and pulsed Doppler imaging. All examinations were performed with an Acuson Sequoia 512 system (Siemens Medical Solution, Mountain View, CA). Transabdominal imaging alone was performed and was diagnostic in all but 4 patients, who required additional endovaginal supplementation. Various transducers ranging from 2.5 to $5.0 \mathrm{Mhz}$ were used for transabdominal imaging. An 6.0 - 8.0 MHz transducer was used for endovaginal imaging.

The diameter of metastasia lesion was measured. Color and pulsed Doppler ultrasound of the metastasia lesion was performed in all cases. The sample volumes were taken from arteries of the metastasia lesion. The spectral waveforms were analyzed for the presence of high-velocity, low-impedance flow. The resistive indices were recorded. In addition, both maternal ovaries were examined. Theca lutein cysts were confirmatory of the hCG level elevation.

Histologic proof of diagnosis was obtained only in 2 cases Choriocarcinoma and 1 case IHM. The diagnosis in the other cases of IHM and Choriocarcinoma was according to the criterion of International Federation of Gynecology \& Obstetrics (FICO, 2000) with the group differentiated on the basis of the latency of symptom appearance after evacuation of the initial affected gestational [1], the hCG level, clinical examination, sonographic findings, and so on. Patients became symptomatic within the subsequent 6 months after curettage of hydatidform molar tissue were stratified into the IHM group. Those who became symptomatic after 12 months of dilation and suction evacuation for the hydatidform mole or after abortion were placed in the choriocarcinoma group.

Once an IHM or choriocarcinoma was diagnosed, all patients were treated with combination chemotherapy 
protocols using fluorouracil, kengshengmycin, nitrocaphane, methotrexate, and etoposide. Patients' follow-up by sonography and serum hCG analysis was attempted in all cases. Similar sonographic criteria were used to diagnose the presence of recurrent disease on patients with persistent or rising serum hCG levels. All treated patients were followed until hCG levels became normal and until the sonographic and Doppler abnormalities disappeared. It was necessary for some cases to have examination by computed tomography and chest radiography

\section{Results}

Metastasia lesion was detected in all 13 cases. 4 women were from choriocarcinoma; 9 were from IHM. All patients had vaginal bleeding clinically. Most had abdominal pain and nausea as well. On physical examination, uterine enlargement was the most common finding, and 7 cases could be touched masses in pelvic cavity by gynecologist. Serum hCG levels were universally elevated in all cases of GTT at initial examination with levels measuring from $500 \mathrm{ng} / \mathrm{ml}$ to $2000 \mathrm{ng} / \mathrm{ml}$. Doppler waveforms showed resistive indices of 0.34 (SD 0.07) for all metastasia lesion. The sonographic and Doppler findings are summarized in Table 1.

In the 13 patients with GTT, 5 cases occurred during the first pregnancy; 4 occurred during the second; 3 occurred during the third; and 1 occurred during the fourth.

In the 4 patients with choriocarcinoam, 1 initially had a hydatidform mole and was treated by dilation and suction evacuation one year ago; and 3 cases occurred after an elective therapeutic abortion. These cases were diagnosed because of having invasive disease within the myometrium, as well as metastasia lesion by follow-up sonographic and Doppler examination. The sites of metastasia lesion were 1 had solid lesion in uterine fundus (Figure 1), 1 had metastasia lesion with reticular structure near uterus (Figure 2), 1 had colonic metastasia lesion with pseudo kidney sign (Figure 3), and 1 had cystic vascular space near the uterus (Figure 4).

\section{Table 1. Summary of sonographic and Doppler findings of Metastasia lesion in 13 cases of GTT.}

\begin{tabular}{|c|c|c|c|c|c|}
\hline \multirow{2}{*}{ Case } & \multirow{2}{*}{ Age } & \multirow{2}{*}{ Diagnosis } & \multicolumn{2}{|c|}{ Metastasia Lesion } & \multirow{2}{*}{ Color Doppler Imaging } \\
\hline & & & Site & Diameter (cm) & \\
\hline 1 & 21 & choriocarcinoma & uterine fundus & $3.1 \times 2.3$ & $\begin{array}{l}\text { solid lesion with well-defined, abundant blood } \\
\text { flow, RI: } 0.29\end{array}$ \\
\hline $2^{*}$ & 28 & choriocarcinoma & near uterus & $4.2 \times 1.8$ & $\begin{array}{l}\text { reticular structure with no well-defined, and } \\
\text { abundant blood flow, RI: } 0.20\end{array}$ \\
\hline $3^{*}$ & 46 & choriocarcinoma & colon & $3.5 \times 2.6$ & pseudo kidney sign \\
\hline 4 & 41 & choriocarcinoma & $\begin{array}{l}\text { cystic vascular space } \\
\text { near the uterus }\end{array}$ & $7.0 \times 4.5$ & abundant blood flow, RI: 0.29 \\
\hline 5 & 31 & IHM & $\begin{array}{l}\text { cystic vascular space } \\
\text { near the uterus }\end{array}$ & $5.5 \times 3.9$ & abundant blood flow, RI: 0.27 \\
\hline 6 & 35 & IHM & $\begin{array}{l}\text { cystic vascular space } \\
\text { near the uterus }\end{array}$ & $3.3 \times 1.4$ & abundant blood flow, RI: 0.25 \\
\hline 7 & 35 & IHM & $\begin{array}{l}\text { cystic vascular space } \\
\text { near the uterus }\end{array}$ & $4.7 \times 4.5$ & abundant blood flow, RI: 0.33 \\
\hline 8 & 33 & IHM & $\begin{array}{l}\text { side wall of pelvic } \\
\text { cavity }\end{array}$ & $4.5 \times 2.7$ & $\begin{array}{c}\text { reticular structure and with vessels more and } \\
\text { wider, RI: } 0.40\end{array}$ \\
\hline 9 & 36 & IHM & $\begin{array}{l}\text { side wall of pelvic } \\
\text { cavity }\end{array}$ & $4.3 \times 1.9$ & $\begin{array}{c}\text { reticular structure and with vessels more and } \\
\text { wider, RI: } 0.35\end{array}$ \\
\hline $10^{*}$ & 43 & IHM & near uterus & $3.1 \times 1.7$ & solid lesion, RI: 0.32 \\
\hline 11 & 26 & IHM & abdominal cavity & $6.0 \times 5.6$ & abundant blood flow, RI: 0.37 \\
\hline 12 & 33 & IHM & colon & $7.1 \times 5.7$ & $\begin{array}{l}\text { colonic wall borden and lesion with well-defined, } \\
\qquad \text { RI: } 0.46\end{array}$ \\
\hline 13 & 35 & IHM & right renal & $9.3 \times 5.6$ & Well-defined and abundant flow, RI: 0.40 \\
\hline
\end{tabular}

Histologic proof. 


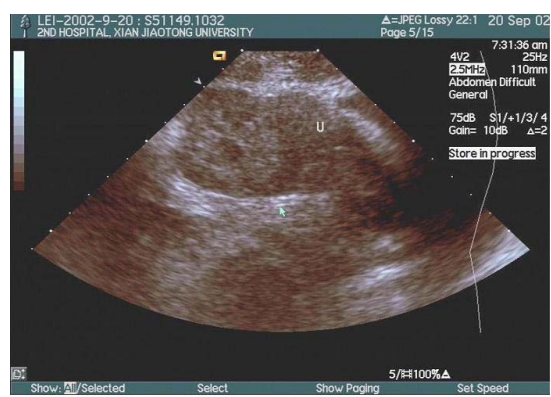

(a)

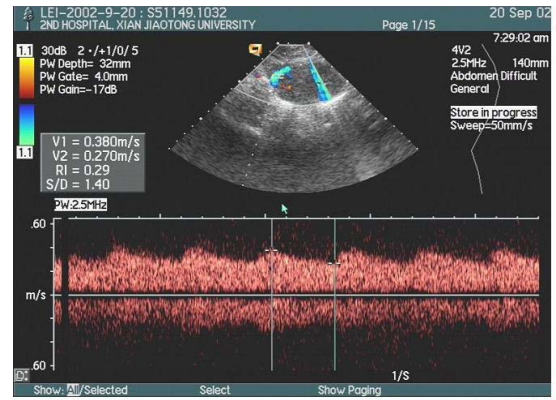

(c)

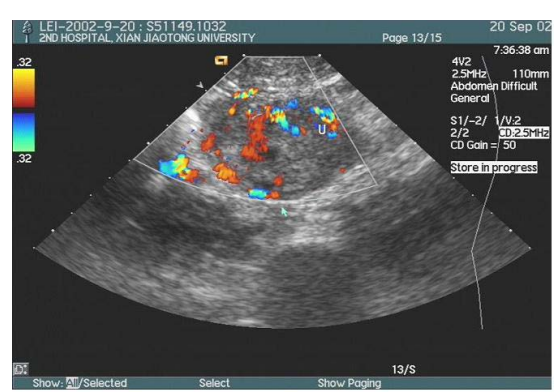

(b)

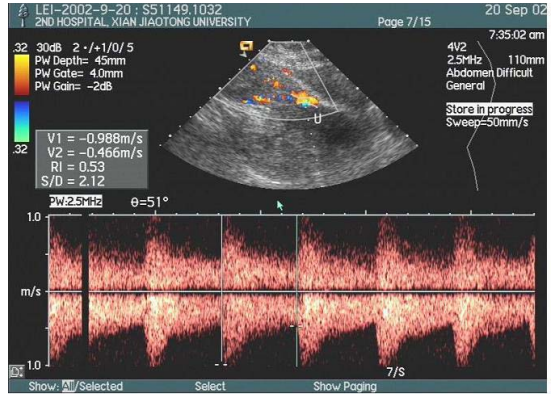

(d)

Figure 1. Metastasia lesion in uterine fundus with a choriocarcinoma in the same patient. (a) Longitudinal sonogram shows a solid lesion in uterine fundus; (b) Color Doppler imaging shows blood flow of the metastasia lesion; (c) Doppler waveform shows arteries flow of in the metastasia lesion with a resistive index of 0.29 ; (d) Following-up sonogram after 6 courses of combination chemotherapy shows the uterine blood flow has became normal, with a resistive index of 0.53 . Now the woman had a baby. U: uterus.

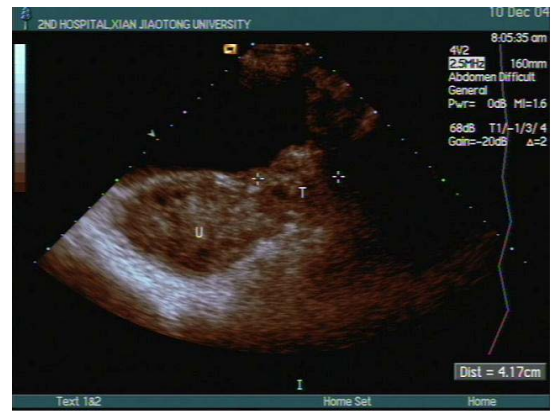

(a)

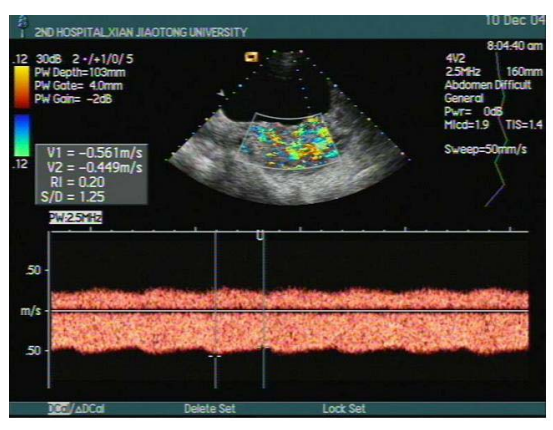

(b)

Figure 2. Metastasia lesion near the uterus with a choriocarcinoma in the same patient. (a) Transverse sonogram shows an irregular lesion with reticular structure near the uterus; (b) Doppler waveform shows arteries flow of the metastasia lesion with a resistive index of 0.20 . U: uterus; T: metastasia lesion.

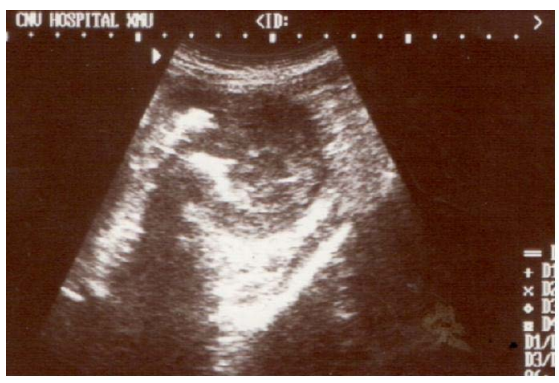

Figure 3. Colonic metastasia lesion with a choriocarcinoma, which had ever treated with combination chemotherapy for three months. Transverse sonogram shows pseudo kidney sign, colonic resection. 


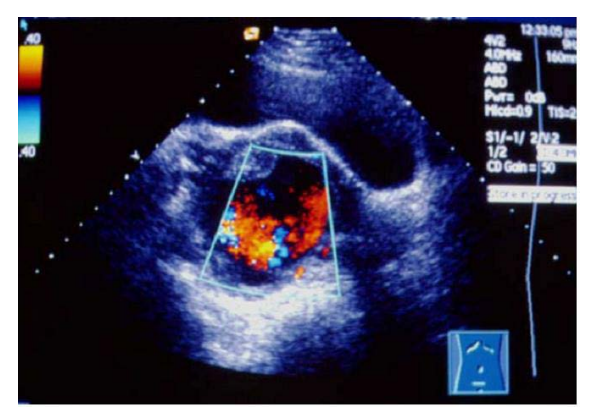

Figure 4. Cystic vascular space near the uterus with choriocarcinoma. Color Doppler imaging shows abundant blood flow in the cystic vascular space.

In addition to vaginal bleeding and abdominal pain, 1 woman with choriocarcinoam had a headache. Distant brain metastasia lesion was diagnosed with the aid of computed tomography.

All 9 patients with IHM initially had a hydatidform mole and were treated by dilation and suction evacuation. These cases were diagnosed as having invasive disease soon after one to three months of the dilation and suction evacuation for the hydatidform mole because the hCG levels failed to normalized. Metastasia lesion was detected by follow-up sonographic and Doppler examination. The sites of metastasia lesion were: 3 had cystic vascular space near the uterus, 2 had side wall metastasia lesion of pelvic cavity with irregular reticular structure (Figure 5), 1 had solid metastasia lesion near uterus, 1 had metastasia lesion of abdominal cavity, 1 had colonic metastasia lesion, and 1 had right renal metastasia lesion with hCG level around 930 ng/L (Figure 6).

In addition to vaginal bleeding and abdominal pain, 5 women with IHM had a cough, and 2 had a headache. Distant pulmonary metastasia lesion and brain metastasia lesion were diagnosed with the aid of computed tomography and chest radiography.

Curative effects were observed in all cases. The case 13, an IHM with renal metastasia lesion, (Figure 6(c)), Following up sonogram after 8 courses of combination chemotherapy showed the renal metastasia lesion did not disappear, but the uterine blood flow became normal, and the blood flow of metastasia lesion disappeared. From then on, the patient had been observing for one and half years. The case 1 , a metastasia lesion in uterine fundus with a choriocarcinoma, (Figure $\mathbf{1}(\mathrm{d})$ ), following up sonogram after 6 courses of combination chemotherapy was found that uterine blood flow became normal with a resistive index of 0.53 . Now the woman had a baby after two years. The case 3 who had colonic resection was diagnosed wrong as tumor at first by ultrasound. Histologic of diagnosis was choriocarcinoma. Besides those, abnormal sonographic and Doppler findings in metastasia disease disappeared when chemotherapy was successful.

\section{Discussion}

Although the incidence of GTT varies considerably in different regions of the world [2], Chinese populations consistently are affected more often than other ethnic groups, and 15\% - 20\% [12] is reported by Beijing Xiehe hospital in China. The reason is not fully understood but may have a genetic basis [13]. Typically, Color Doppler sonography shows low-resistance arterial flow $(\mathrm{RI}<0.4)$ invades into the uterine myometrium, consistant with a markedly elevated serum hCG level [2]. It is useful in diagnosing GTT and having replaced angiography of the uterus [14]-[18]. Although there have been many methods used for diagnosing GTT, the metastasia lesion in abdominopelvic cavity by using ultrasound is still rarely reported [19]-[21]. We have analyzed metastasia lesion of 13 GTT in abdominopelvic cavity and found it is helpful in therapy and prognosis.

Trophoblastic tumor derives from gestation, so we call it gestation trophoblastic tumor. Of GTT, the distant metastasis always occurs in patient with GTT, no matter the extent of malignancy. The trophoblastic tissue invades the maternal vascular system and is transported to not only local site as pelvis but also a farther site, such as kidney, colon, lung, brain, and so on. In our group, 13 cases with GTT were found to have metastsatic disease. The sites of metastasia lesion were: 4 had cystic vascular space near the uterus, 2 had colonic metastasia lesion, 2 had side wall metastasia lesion of pelvic cavity, 1 had right renal metastasia lesion, 1 had metastasia lesion of abdominal cavity, and 3 had metastasia lesion near uterus. The diameter of metastasia lesion was different from $1.4 \mathrm{~cm}-9.3 \mathrm{~cm}$. So color Doppler sonography not only has become the standard in comfirming the diagnosis, but also in finding metastasia lesion. But color Doppler sonography could not find pulmonary or brain metastasia lesion, with the aid of computed tomography and chest radiography, the other metastasia lesion may be detected. 


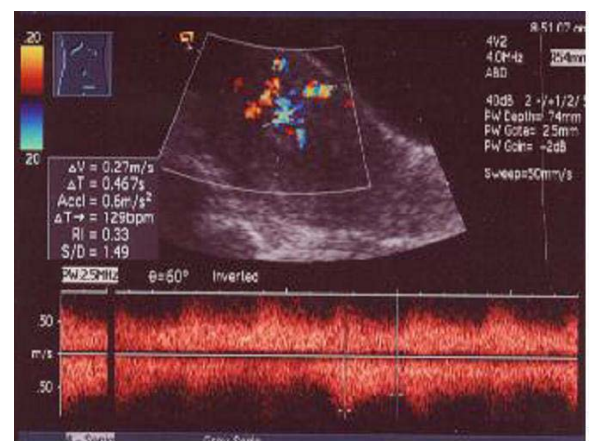

(a)

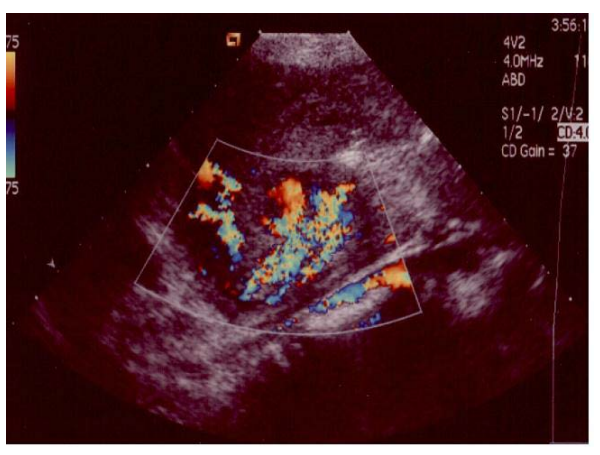

(b)

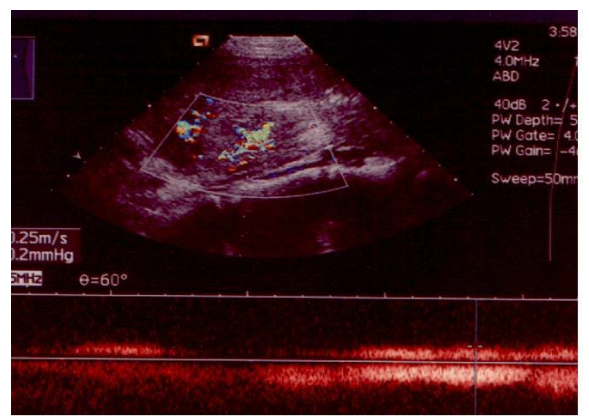

(c)

Figure 5. Side wall metastasia lesion of pelvic cavity with an IHM in the same patient. (a) Doppler waveform shows arteries flow of invasive disease in the myometrium with a resistive index of 0.33; (b) Color Doppler imaging shows side wall metastasia lesion of pelvic cavity; (c) Doppler waveform shows venous flow in the side wall metastasia lesion.



(a)

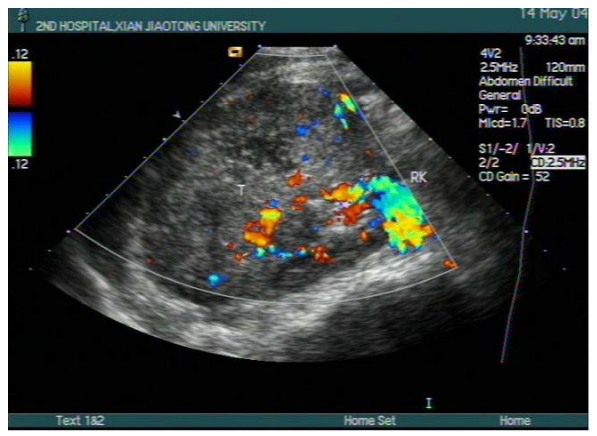

(b)



(c)

Figure 6. Right renal metastasia lesion with an IHM in the same patient. (a) Doppler waveform shows arteries flow of invasive disease in the myometrium with a resistive index of 0.28; (b) Color Doppler imaging shows right renal metastasia lesion; (c) After 8 courses of combination chemotherapy shows the flow of right renal metastasia lesion disappear. 
Although pathologic characteristics between IHM and choriocarcinoma are different, the results are the same, uterine muscular layer and vessel system are invaded by abnormal trophoblastic proliferation histologically, which always cause hemorrhage and necrosis [22]. So the metastasia lesion of IHM shows the same imaging as choriocarcinoma's. And the reticular structure, the cystic vascular space and solid lesion may be showed by ultrasound.

In this study, Doppler sonogram shows low-impedance flow in cases of distant metastasia lesion with resistive indices of less than 0.4. Presumably, as well as myometeial invasion, this indicates a greater degree of vascular invasion by trophoblastic tissue at the time of diagnosis by the invasive entities [10]. The causation of lowimpedance flow is the form of arteriovenous fistula. Normal uterine venous blood could flow backwards without any blocking due to lack of venous valve in uterine vein, which allow the abnormal trophoblastic tissue of GTT keep invading from veins to arteries [22]. So, the characteristic high-flow, low-impedance arterial blood flow pattern is formed. That is why local and distant metastases of GTT are so common.

In addition to serum hCG levels, Doppler sonogram is helpful in detecting disease recurrence and evaluating the efficacy of treatment [21]. The characteristic high-flow, low-impedance arterial blood flow pattern of metastasia lesion of GTT allows Doppler studies to aid in this evaluation, as well as myometeial invasion. In our group, the case 2 and the case 10 had hysterectomy because their age were older and chemotherapy effect were not better. The case 3 with colonic metastasia lesion had colonic resection. After operation, they were treated with combination chemotherapy like other cases. As we have seen, except an IHM with renal metastasia lesion having been observed, abnormal sonographic and Doppler findings in other metastasia disease disappeared when chemotherapy was successful. The greatest utility of the Doppler flow studies was in their ability to show the presence of disease response to chemotherapy.

A case was diagnosed wrong as colonic tumor at first (the case 3). It was colonic metastasia lesion with a choriocarcinoma showing pseudo kidney sign. The case had been treated with combination chemotherapy for three months because of choriocarcinoma before colonic resection. From this case, we can learn much. The better therapeutic strategy in the future that we should take serious consideration is to detect metastasia lesion except uterus and ovary.

In China, nearly pelvic sonography is performed by the transabdominal approach. Endovaginal supplementation is used only in occasional cases when the transabdominal approach proved confusing or nondiagnostic. In this group, endovaginal sonography was performed in only 4 patients. Even then, the sonographic and Doppler imaging was thought to be adequate for diagnosis. In this study, the diagnosis of IHM or choriocarcinoma was proved histologically in three cases. In the other cases, the diagnosis was presumed on the basis of clinical appearance and the latency of symptom onset. According to criterion referred, of the 13 patients with GTT, 9 had an invasive hydatidiforn mole (IHM), 4 had choriocarcinoma. Although the assigned diagnosis was the most likely, the accuracy was not perfect. Especially no cases of metastasia disease with placental site trophoblastic tumor were reported in this study and in the articles about 12-year experience in China had been published [10] [11]. A placental site trophoblastic tumor is derived from extravillous trophoblastic cells of the placental bed. Patients generally have either heavy irregular bleeding or amenorrhea [23]. It tends to be high-risk metastatic disease because of being resistant to chemotherapy, so pulmonary, ovarian, subaponeurotic, lymph lesion, brain, and adrenal metastasis are so common [24]-[29]. This disease often needs hysterectomy for treatment.

\section{References}

[1] Xie, X. (2003) Gestation Trophoblastic Disease. In: Le, J., Ed., Gynecology and Obstetrics, 6th Edition, People’s Sanitation Publish Company, Beijing, 319-328.

[2] Fox, H. (1991) Female Reproductive System. In: Anderson, M.C., Ed., Systemic Pathology, Volume 6, 3rd Edition, Churchill Livingstone, New York, 435-445.

[3] Xu, X.J., Lou, F.L., Zhang, M.M., Pan, Z.M. and Zhang, L. (2007) Usefulness of Low-Dose CT in the Detection of Pulmonary Metastasis of Gestational Trophoblastic Tumours. Clinical Radiology, 62, 998-1003. http://dx.doi.org/10.1016/j.crad.2007.03.009

[4] Suprasert, P., Eua-Throngchit, J., Srisomboon, J., Charoenkwan, K., Siriaree, S. and Phongnarisorn, C. (2005) Radiologic Features and Treatment Outcomes of Pulmonary Metastasis in Gestational Trophoblastic Neoplasia. Journal of the Medical Association of Thailand, 88, 875-880.

[5] Park, S.G., Chang, J.Y., Kim, S.H. and Bang, D. (2005) Cutaneous Metastasis of Choriocarcinoma: A Case Report. Journal of Korean Medical Science, 20, 683-686. http://dx.doi.org/10.3346/jkms.2005.20.4.683

[6] Zhang, Y., Xiang, Y., Ren, T., Wan, X.R. and Yang, X.Y. (2005) Study on the Indication of Surgical Resection of Pul- 
monary Metastasis of Malignant Trophoblastic Tumor. Chinese Journal of Obstetrics and Gynecology, 40, 83-86.

[7] Perroni, D., Grecchi, G.L., La Ciura, P. and Landoni, F. (1993) Right Ventricular Metastasis from Choriocarcinoma: Report of a Rare Case and Review of the Literature. European Journal of Surgical Oncology, 19, 378-381.

[8] Yamamoto, N., Shinoda, I., Takeuchi, T., Matsuda, S., Kuriyama, M., Ban, Y. and Kawada, Y. (1988) A Case of Pure Choriocarcinoma of the Testis with Cerebellar Metastasis. Hinyokika Kiyo/Acta Urologica Japonica, 34, 1475-1478.

[9] Ren, T., Xiang, Y., Yang, X.Y., et al. (2000) Treatment and Prognosis Gestation Trophoblastic Tumor in Bladder Metastasis. Chinese Journal of Obstetrics and Gynecology, 1, 36-38.

[10] Zhou, Q., Lei, X.Y., Xie, Q. and Cardoza, J.D. (2005) Sonographic and Doppler Imaging in the Diagnosis and Treatment of Gestational Trophoblastic Disease: A 12-Year Experience. Journal of Ultrasound in Medicine, 24, 15-24.

[11] Lei, X.Y., Xi, M.Y., Liu, J., et al. (1996) Study of Trophoblastic Disease by Color Doppler Flow Imaging. Journal of Xi'an Medical University, 17, 328-329.

[12] Song, H.Z., Wu, B.Z. and Tang, M.Y. (1983) The Diagnosis and Therapy in Trophoblastic Tumor. People’s Sanitation Publish Company, Beijing, 110-115.

[13] Ho, H.N., Gill III, T.J., Klionsky, B., Ouyang, P-C., Hsieh, C-Y., Seski, J., et al. (1989) Differences between White and Chinese Populations in Human Leukocyte Antigen Sharing and Gestational Trophoblastic Tumors. American Journal of Obstetrics and Gynecology, 161, 942-948. http://dx.doi.org/10.1016/0002-9378(89)90758-8

[14] Chan, F.Y., Chau, M.T., Pun, T.C., Lam, C., Ngan, H.P. and Wong, R.L. (1995) A Comparison of Color Doppler Sonography and the Pelvis Arteriogram in Assessment of Patients with Gestational Trophoblastic Disease. British Journal of Obstetrics and Gynaecology, 102, 702-725.

[15] Xiang, Y., Yang, X., Yang, N. and Song, H. (1998) A Comparative Study of Transvaginal Ultrasonograhy and Pelvic Arteriogram in Assessment of Patients with Gestational Trophoblastic Tumour. Chinese Medical Sciences Journal, 13, 45-48.

[16] Taylor, K.J., Schwartz, P.E. and Kohorn, E.I. (1987) Gestational Trophoblastic Neoplasia: Diagnosis with Doppler US. Radiology, 165, 445-448. http://dx.doi.org/10.1148/radiology.165.2.2821575

[17] Carter, J., Fowler, J., Carlson, J., Saltzman, A., Byers, L., Carson, L., et al. (1993) Transvaginal Color Flow Doppler Sonography in the Assessment of Gestational Trophoblastic Disease. Journal of Ultrasound in Medicine, 12, 595-599.

[18] Flam, F. (1994) Colour Flow Doppler for Gestational Trophoblastic Neoplasia. European Journal of Gynaecological Oncology, 15, 443-448.

[19] Sui, X.F., Li, M.G., Zhao, Z.H. and Ye, X.J. (2006) Value of Color Doppler Flow Imaging in Malignant Gestational Trophoblastic Tumors. Chinese Journal of Ultrasound Diagnosis, 7, 446-448.

[20] Shi, Y.F., Ye, D.F., Lu, W.G., et al. (2001) Dynamic Observation of Uterine Lesion Gestational Trophoblastic Tumor with Color Doppler Blood Flow Imaging. Journal Practical Obstetrics and Gynecology, 17, 90-92.

[21] Hong, Y., Zhong, W.J. and Fu, X.Y. (2004) Value of Transvaginal Color Doppler Flow Imaging in Diagnosing Choriocarcinoma. Chinese Journal of Ultrasound Diagnosis, 5, 193-195.

[22] Chen, Z.N. (1992) Gestational Trophoblastic Diseases. In: Du, X.G., Ed., Pathology of Obstetrics and Gynecology, Shanghai Medical University, Shanghai, 50-57.

[23] Jauniaux, E. (1997) Diagnosis and Follow-Up of Gestational Trophoblastic Disorders. In: Rumak, C.M., Wilson, S.R. and Charboneau, J.W., Eds., Diagnostic Ultrasound, 2nd Edition. Mosby Co., St. Louis, 847-856.

[24] Cole, M.E., Broaddus, R., Thaker, P., Landen, C. and Freedman, R.S. (2008) Placental-Site Trophoblastic Tumors: A Case of Resistant Pulmonary Metastasis. Nature Clinical Practice Oncology, 5, 171-175. http://dx.doi.org/10.1038/ncponc1042

[25] Milingos, D., Doumplis, D., Savage, P., Seckl, M., Lindsay, I. and Smith, J.R. (2007) Placental Site Trophoblastic Tumor with an Ovarian Metastasis. International Journal of Gynecological Cancer, 17, 925-927. http://dx.doi.org/10.1111/j.1525-1438.2007.00881.x

[26] Aoki, Y., Kase, H., Kashima, K., Yahata, T. and Tanaka, K. (2005) Placental site Trophoblastic Tumor Presenting as Subaponeurotic Metastasis. International Journal of Gynecological Cancer, 15, 385-388. http://dx.doi.org/10.1111/j.1525-1438.2005.15235.x

[27] Metindir, J., Pak, I., Ozdilekcan, C. and Eren, E. (2005) Chemoresistant Placental Site Trophoblastic Tumor with Hilar Lymph Lesion Metastasis: An Unusual Site of Involvement. Gynecologic Oncology, 96, 552-555. http://dx.doi.org/10.1016/j.ygyno.2004.10.030

[28] Guvendag, G.E.S., Guven, S., Esinler, I., Ayhan, A., Kucukali, T. and Usubutun, A. (2004) Placental site Trophoblastic Tumor in a Patient with Brain and Lung Metastases. International Journal of Gynecological Cancer, 14, 558-563. http://dx.doi.org/10.1111/j.1048-891x.2004.014322.x

[29] Bruni, R., Nigita, G., Pagani, G.B.V., Petrocca, S. and Terenzi, A. (2003) Adrenal Metastatic Placental Site Trophoblastic Tumor. Case Report. Chirurgia Italiana, 55, 257-260. 